\title{
Uma revisão sobre a adoção da TRIZ nas metodologias de desenvolvimento de novos produtos
}

\section{A review about TRIZ employment on new product development methodologies}

FERNANDES, Eduardo da Cunda Fernandes; Bacharel; UFRGS

eduardo.cunda@ufrgs.br

TEIXEIRA, Fábio Gonçalves; Doutor; UFRGS

fabiogt@ufrgs.br

PACHECO, Joyson Luiz; Doutor; UFRGS

joyson@mecanica.ufrgs.br

\section{Resumo}

Considerando-se a grande utilidade da TRIZ, e as atuais tendências mundiais, é esperado que seja verificado o uso desta ferramenta especialmente na elaboração de novos produtos, em particular na área de produtos sustentáveis e ecológicos, e de integração a metodologias existentes no mercado. Neste contexto o presente trabalho visa compilar as principais áreas de pesquisa e tendências de utilização da TRIZ, além de indicar as suas ferramentas utilizadas no desenvolvimento de novos produtos (DNP), abordando especialmente seu emprego em termos de metodologia e integração a processos de projeto de produtos. Assim, apresenta-se o método TRIZ e suas principais ferramentas e fundamentos. Na sequência são apresentados trabalhos encontrados acerca do uso da TRIZ, enfocando nos casos onde há uma integração entre metodologias. Enfim, este trabalho conclui ressaltando os pontos fracos e fortes da TRIZ, bem como apresentando um resumo das linhas de pesquisa encontradas de acordo com a visão deste autor.

Palavras Chave: TRIZ, DNP, Eco-Design.

\begin{abstract}
Considering the TRIZ great utility, and current world trends, it is expected that its tools use will be verified especially in the elaboration of new products, in particular on the creation of sustainable and ecological products, and its integration with existing methodologies in the market. In this context, the present work aims to compile the main research areas and trends of use of TRIZ, in addition to relating its used tools in new products development (NPD), especially addressing its employment in terms of methodology and integration to products design processes. Thus, the TRIZ method and its main tools and fundamentals are presented. In the sequence are presented works about the use of TRIZ, focusing on cases where there's integration with NPD methodologies. Finally, this paper concludes by highlighting the weaknesses and strengths of TRIZ, as well as presenting a summary of research lines found according to this author's vision.
\end{abstract}

Keywords: TRIZ, NPD, Eco-Design. 


\section{Introdução}

A TRIZ (Теория решения изобретательских задач em russo, ou em português Teoria da Resolução de Problemas Inventivos) foi desenvolvida por G. Altshuller (1984) durante os tempos da URSS com objetivo de resolver problemas de inovação técnica (LÓPEZ; ALMEIDA; ARAUJOMOREIRA, 2005), resultado da análise de mais de 2 milhões de patentes, o que representava cerca de $10 \%$ de todas as patentes do mundo à época (STAROVOYTOVA, 2015). O trabalho de G. Altschuller se difundiu pelo mundo como resultado da imigração de seus alunos devido a introdução da glasnost (STAROVOYTOVA, 2015), uma política que visava um processo de abertura política.

Na sequência da história, e especialmente a partir dos anos 2000, observou-se tecnologias de informação e comunicação se disseminarem, resultando em grande ampliação do alcance das pesquisas, ainda mais estimulada pela globalização. É nesse período que a TRIZ começou a atrair maior atenção, sendo empregada com diversos objetivos e em diversas áreas, e consequentemente comprovando sua grande utilidade. Dentre suas diversas aplicações, encontra-se a área de gerenciamento, da educação e no desenvolvimento e integração com metodologias de desenvolvimento de novos produtos (CHECHURIN, 2016). Na área de gerenciamento, geralmente encontra-se pesquisas integrando-se TRIZ e a Teoria das Restrições; na educação é possível encontrar trabalhos realizados com objetivo de estimular a criatividade em alunos de engenharia (CHANG et al., 2016), física, química, educação e ciências sociais, especialmente com a utilização dos 40 princípios inventivos (LÓPEZ; ALMEIDA; ARAUJO-MOREIRA, 2005).

Em termos de desenvolvimento de produtos, porém, encontra-se a maior parte dos artigos analisados durante esta pesquisa. De modo geral, é possível verificar especialmente o enfoque a nível de desenvolvimento de produtos sustentáveis e ecologicamente amigáveis (BRAD et al., 2016; RUSSO; RIZZI; MONTELISCIANI, 2014; RUSSO; REGAZZONI; MONTECCHI, 2011; VINODH; KAMALA; JAYAKRISHNA, 2014; VINODH; RATHOD, 2010; YANG; CHEN, 2011, 2012). Outras pesquisas abordam a integração das matrizes de Desdobramento da Função Qualidade (QFD) e TRIZ como ponto de partida, uma vez que a primeira apresenta especial enfoque nas necessidades dos usuários, enquanto a segunda é excelente ferramenta de inovação técnica (CHEN; CHEN, 2011; HUANG, 2013; PELT; HEY, 2011; WANG; YEH; CHU, 2016; YAMASHINA; ITO; KAWADA, 2000).

Considerando que a TRIZ foi elaborada como resultado da pesquisa de patentes, é esperado que seja verificado seu uso especialmente na elaboração de novos produtos, seguindo a demanda da tendência sustentável e ecológica e de enfoque nos usuários a nível de mercado e de concorrência, além da integração a metodologias já consagradas pela industrias. Neste contexto este trabalho busca apresentar um resumo com os resultados encontrados na pesquisa, visando compilar as principais áreas de pesquisa e tendências de utilização da TRIZ, além de suas ferramentas mais utilizadas no desenvolvimento de novos produtos (DNP), abordando especialmente sua adoção a nível de metodologias e integração a processos de projeto de produtos. Além disso, para facilitar a compreensão do que será apresentado, e visando promover a TRIZ, este trabalho previamente apresenta uma síntese acerca de sua teoria e principais ferramentas.

Neste artigo, portanto, apresenta-se uma revisão acerca das pesquisas sobre a Teoria da Resolução de Problemas Inventivos, onde segue-se a seguinte estrutura: na seção 2 é introduzido o método TRIZ e suas principais ferramentas e fundamentos; na seção 3 é apresentada a metodologia adotada para esta pesquisa; na seção 4 são apresentados os principais estudos encontrados quanto a utilização dos métodos da TRIZ; na seção 5 são desenvolvidos algumas observações e comentários 
relativos a pesquisa desenvolvida bem como as conclusões da presente pesquisa; por fim a seção 6 apresenta sugestões para futuras pesquisas.

\section{Teoria da Resolução de Problemas Inventivos - TRIZ}

Buscando resolver problemas recorrentes do método de tentativa e erro, G. Altshuller foi o pioneiro a apresentar a Teoria da Resolução de Problemas Inventivos (ALTSHULLER, 1996; YANG; CHEN, 2011), posteriormente apresentada em 1965 após a análise de mais de 400,000 patentes do mundo todo (CHEN; CHEN, 2011; CONG; TONG, 2008). Nos últimos 50 anos podem ser contabilizadas as análises de mais de dois milhões de patentes, das quais foi possível concluir três resultados decorrentes do processo de inovação nas ciências e industrias (HUANG, 2013; LÓPEZ; ALMEIDA; ARAUJO-MOREIRA, 2005), (i) os problemas e padrões se repetem, (ii) os padrões de evolução técnica se repetem e (iii) as inovações são resultado de efeitos científicos de campos diferentes dos quais são desenvolvidos.

O resultado da análise das patentes são as ferramentas desenvolvidas por Altshuller e seus pesquisadores (ALTSHULLER, 1996, 1984; ALTSHULLER; SHULYAK; RODMAN, 1997), tais como as 76 soluções-padrão, a matriz de análise de contradição, os 40 princípios inventivos, os 39 parâmetros de engenharia, etc (CHEN; CHEN, 2011). Através dos dispositivos apresentados, a TRIZ é capaz de apresentar um modelo sistemático, estruturado e racional para o pensamento criativo eficaz na geração de soluções para problemas técnicos, diminuindo o risco de insucesso no desenvolvimento de novos produtos.

Nos tópicos seguintes são apresentados os principais fundamentos e ferramentais utilizados nos trabalhos pesquisados.

\subsection{Níveis de Criatividade}

Em seus trabalhos, Altshuller elaborou uma classificação de criatividade a cada patente. São 5 os níveis, sendo que os dois primeiros apresentam inovações muito pequenas. Exemplo de inovação nível 1 é a alteração de um desenho, como por exemplo, adaptar um relógio de bolso em um relógio de pulso. Já uma inovação de nível 2 corresponde a uma melhoria, como por exemplo, a adição de uma nova função como o despertador a um relógio (LÓPEZ; ALMEIDA; ARAUJOMOREIRA, 2005).

Os demais níveis apresentam alguma real inovação. O nível 3 caracteriza-se por uma melhoria, capaz de resolver uma contradição, utilizando métodos de outras áreas afins ao do produto em desenvolvimento. Um exemplo é o relógio digital, que apresenta utilização de conhecimentos de eletrônica. O nível 4 apresenta melhorias, geralmente de cunho científico, capazes de apresentar um novo princípio à principal função de um produto. Um exemplo é o caso de relógios atômicos. O maior nível da classificação é o 5, e abriga raras descobertas científicas tais como, por exemplo, os raios $X$, a sequência do DNA e a supercondutividade (LÓPEZ; ALMEIDA; ARAUJO-MOREIRA, 2005). Os 8 Princípios, ou Tendências, da Evolução Tecnológica

Ao analisar de milhares a milhões de patentes Altshuller percebeu que havia padrões de evolução tecnológica capazes de descrever o progresso de tecnologias voltadas à resolução de problemas. A partir disso, foram identificados os seguintes oito padrões de evolução tecnológica apresentados no Quadro 1 (ALTSHULLER, 1984; IDEATION INTERNATIONAL INC., 1999; LEON, 2006; RUSSO; REGAZZONI; MONTECCHI, 2011; SALAMATOV, 1999). 
Quadro 1 - Padrões de evolução tecnológica identificados por Altschuller.

\section{Os 8 padrões de evolução tecnológica}

1. A tecnologia cumpre ciclo no qual, em analogia, nasce, cresce, atinge maturidade e depois entra em declínio;

2. Os sistemas evoluem de modo a atingirem seus maiores graus de idealidade (ver capítulo 2.3);

3. As partes do sistema evoluem de modo desigual, gerando problemas de contradição e resultando em problemas inovativos a serem resolvidos;

Os sistemas evoluem aumentando seu dinamismo/flexibilidade e controlabilidade. Isso significa que os

4. sistemas passam a ser mais fáceis de serem controlados, ao mesmo tempo que aumenta a flexibilidade de suas aplicações;

Aumento da complexibilidade seguido de simplificação através da integração das partes, i.e., há uma tendência

5. de aumentar a quantidade de funções de um sistema - aumentando seu nível de complexibilidade - e posteriormente evoluírem de modo a apresentarem sistemas mais simples com estas mesmas funções integradas;

6. A evolução ocorre combinando e retirando elementos visando melhorar desempenho ou compensar efeitos indesejados;

7. Transição de macrosistemas para microssistemas através de campos de energia visando melhor desempenho ou controle do sistema;

8. Aumento da automação dos sistemas com consequente decréscimo do envolvimento humano.

Fonte: Altschuller (1984), Ideation International Inc. (1999), Leon (2006); Russo, Regazzoni e Montecchi (2011) e Salamatov (1999).

\subsection{Recursos}

Um dos fundamentos da TRIZ trata do mapeamento e máximo proveito dos recursos disponíveis. Para Mishra (2013) os recursos podem ser divididos em (i) materiais, tais como matéria prima, maquinário, animais, plantas, capital financeiro, etc, (ii) campo ou energia, como eletricidade, luz, campos magnéticos e eletromagnéticos, etc, (iii) informativos, como informações de patentes, internet, livros, etc, (iv) espaciais, em termos de espaço disponível para uso, operação, etc, (v) temporais, e (vi) funcionais, como a capacidade de um sistema de realizar funções adicionais. Assim, pode-se compreender que os recursos são quaisquer meios, seja físico, abstrato, visível ou mesmo invisível, que podem ser utilizados no desenvolvimento e estabelecimento de um sistema.

Existem duas principais teorias sobre a utilização dos recursos. Uma delas refere-se ao uso da menor quantidade de recursos, mais baratos, de fácil acesso e que causem baixo impacto nos custos. A outra trata do uso dos recursos indesejados, que causam efeitos negativos, cujo emprego, porém, visa torna-los bons e úteis. Esta última costuma ser a mais utilizada por apresentar resultados que conduzem ao desenvolvimento de produtos revolucionários (MISHRA, 2013).

\subsection{Funcionalidade e a Lei da Idealidade}

O objetivo no desenvolvimento de qualquer sistema ou produto é fornecer alguma função. Para tal finalidade, porém, é necessário que sejam implementados os devidos mecanismos. A lei da idealidade busca compatibilizar essa questão com vistas na diminuição do sistema, barateamento do custo, menor uso de recursos e energia, diminuir volume. Assim, a idealidade é um reflexo da máxima otimização do uso dos recursos disponíveis para um sistema (LANGEVIN; TECHNICAL INNOVATION CENTER INC., 2013; LÓPEZ; ALMEIDA; ARAUJO-MOREIRA, 2005).

Dadas as definições de Idealidade e Recursos, pode-se afirmar que os recursos são a base do desenvolvimento das funções de um sistema, e assim é comum ser adotada a Eq. 1 como forma de 
medição da idealidade de um sistema. Esta equação deixa claro que o consumo de recursos cujos efeitos são negativos devem ser minimizados na busca por um produto ideal (MISHRA, 2013).

$$
\text { Idealidade }=\frac{\text { Boas propriedades e funções úteis }}{\text { Funções prejudicias+Custo }} \quad \text { (Equação 1) }
$$

\subsection{A Matriz de Contradições e os $\mathbf{3 9}$ Parâmetros de Engenharia e os $\mathbf{4 0}$ Princípios Inventivos}

A busca pela idealidade torna-se complexa quando busca-se otimizar as funções de determinado sistema, uma vez que pelo menos um conflito existe em problemas comuns de projetos de produtos. Contradições ocorrem quando busca-se melhorar parâmetros ou características, o que naturalmente causa efeitos negativos em outros parâmetros ou características (SAVRANSKY, 2000). Com objetivo de resolver esses problemas Altshuller resumiu 40 princípios inventivos e 39 parâmetros de engenharia que deram origem a matriz de contradição. $O$ objetivo da matriz é auxiliar a identificar rapidamente quais princípios inventivos podem ser adotados para resolver problemas de contradição de acordo com os parâmetros envolvidos (LIU; CHENG, 2016).

A matriz de contradições pode ser entendida como dividida em dois eixos: $x$ e $y$. $O$ eixo $x$ apresenta os parâmetros que se busca melhorar, e o eixo y indica os parâmetros cujos resultados são indesejados. No campo em que cada um destes parâmetros é identificado e relacionado, podese verificar quais os princípios inventivos são sugeridos (PIMENTEL, 2013).

Apesar de ser a ferramenta mais encontradas nas pesquisas relacionadas à TRIZ, existem outras ferramentas, tais como a Análise Campo-Substância, as 76 Soluções-Padrão e o ARIZ (acrônimo russo de Алгоритмы решения изобретательских задач, em português Teoria da Resolução de Problemas Inventivos).

\subsection{Análise Campo-Substância e as 76 Soluções-Padrão}

Uma das ferramentas originais da TRIZ, a análise campo-substância busca modelar problemas focalizados em sistemas complexos ou em parte de um sistema, sendo possível identificar problemas existentes. Durante a criação, a modelagem de sistemas é realizada em função de suas substâncias (objetos ou atuadores) e forças (ou campos) atuantes, onde a função é resultado de um objeto, causado por outro objeto através da aplicação de alguma forma de campo ou energia (CHEN; CHEN, 2011; PIMENTEL, 2013).

Em conjunto com a aplicação do algoritmo de identificação das relações e problemas são usadas as 76 soluções-padrão elaboradas por Altshuller. Esta lista apresenta uma "descrição concisa para a situação genérica e a descrição das restrições" (PIMENTEL, 2013). As soluções são organizadas em 5 categorias como apresentadas no Quadro 2 (DOMB; MILLER; MACGRAN, 1999).

Quadro 2 - As 5 categorias para organização das 76 soluções-padrão de Altshuller

Categorização das 76 soluções-padrão de Altshuller

1. Melhorar o sistema com nenhuma ou pequenas mudanças (13 soluções padrão);

2. Melhorando o sistema alterando o sistema (23 soluções padrão);

3. Transições de sistema (6 soluções-padrão);

4. Detecção e medição (17 soluções padrão);

5. Estratégias para simplificação e melhorias (17 soluções-padrão).

Fonte: adaptado de Domb, Miller e Macgran (1999) 


\subsection{Algoritmo da Resolução de Problemas Inventivos - ARIZ}

Em 1952, Altshuller desenvolveu um passo-a-asso como uma espécie instruções para inventores, ao qual deu o nome de ARIZ (ALTSHULLER, 1984) em 1970. Através deste algoritmo é possível identificar contradições e soluções inventivas, cujos passos podem ser geralmente resumidos a (i) formulação do problema, (ii) transformação deste problema em um modelo, (iii) análise do modelo, (iv) resolução das contradições e (v) formulação da solução ideal.

\subsection{Método da TRIZ}

O procedimento para resolução de problemas inventivos, conforme a TRIZ, apresenta os seguintes passos: inicialmente identificar o problema inventivo, seguido por uma abstração do problema - ou reformulação - que se relacione com um problema TRIZ para, então, utilizar as devidas ferramentas e encontrar uma solução que permitirá uma tradução para a resolução do problema inicial (LÓPEZ; ALMEIDA; ARAUJO-MOREIRA, 2005; YANG; CHEN, 2011).

\section{Metodologia da Pesquisa}

Este trabalho realiza uma revisão de artigos científicos que integram a TRIZ na resolução de problemas no DNP à nível de metodologias. Uma das metas é incentivar o uso das ferramentas da TRIZ, uma vez que, como pôde ser verificado e comprovado nos trabalhos revisados, apresentam resultados muito eficientes na resolução de problemas inventivos.

Assim, esta é uma pesquisa de caráter qualitativo e exploratório que visa compilar a utilização da TRIZ como uma ferramenta que auxilia e incrementa metodologias de DNP visando sua melhor eficiência. A base de dados utilizada foi a Scopus, sendo os resultados entre os anos 2000 e 2016 considerados como relevantes. Foram diversas as combinações de strings utilizadas, dentre as quais todas relacionam-se com "TRIZ", "design de novos produtos" e "aplicação". Um outro filtro adotado foi a remoção de artigos que utilizavam a TRIZ individualmente na solução de problemas inventivos, bem como artigos que se referiam a áreas alheias ao desenvolvimento de novos produtos, tais como, por exemplo, educação e medicina.

Os resultados obtidos desta busca foram agrupados de acordo com as metodologias com as quais foi proposta a integração da TRIZ. Dentro deste agrupamento, buscou-se a apresentação das proposições metodológicas em ordem cronológica. Assim evita-se a mistura dos resultados encontrados, bem como mantém a ordem de desenvolvimento e aparecimento das novas proposições encontradas.

\section{A Integração da Triz nas Metodologias de Desenvolvimento de Novos Produtos}

O maior destaque apresenta-se na adoção da TRIZ no desenvolvimento de produtos sustentáveis e ecologicamente amigáveis, sendo que inclusive um framework derivado da matriz de contradição da TRIZ foi desenvolvido por pesquisadores, o iTree (RUSSO; RIZZI; MONTELISCIANI, 2014). Além desta linha ligada à um design 'verde', também são encontradas linhas de pesquisa relacionada ao DNP, às quais algumas ferramentas da TRIZ foram integradas a outros modelos de projeto de produtos como, por exemplo, o QFD e o DFSS (Quality Function Deployment e Design for Six Sigma respectivamente). Além destas linhas de pesquisa, também podem ser encontradas outras aplicações da TRIZ, bem como na educação. Estes, porém, não são abordados neste trabalho, ainda que demonstrem potencial e diversas possibilidades de emprego da TRIZ e de suas ferramentas.

Na sequência deste capítulo são apresentadas sínteses dos trabalhos revisados, e que foram 
divididos em 3 partes: TRIZ e o Design Centrado no Ser Humano é uma introdução à linha de pesquisa que une a TRIZ ao projeto de produtos direcionados ao consumidor; TRIZ e o Método de Desdobramento da Função Qualidade, que desenvolve mais informações acerca das metodologias e da importância de integrar a TRIZ à métodos centrados nos usuários, tais como a QFD; e por fim, TRIZ, o desenvolvimento de produtos ecológicos, CBR e a ECQFD, uma linha que apresentou a maior quantidade de artigos encontrados, evidenciando a importância e a tendência no desenvolvimento de produtos sustentáveis e ecologicamente amigáveis.

\subsection{TRIZ e o Design Centrado no Ser Humano}

Em seu trabalho, Pelt e Hey (2011) destacam que o DCSH apresenta uma abordagem pouco estruturada, com pouco apoio ao processo de geração de soluções. Assim, nesta questão fica evidente que a TRIZ apresenta o complemento necessário capaz de alavancar o sucesso no DNP através de uma exploração mais robusta do espaço de soluções. Na abordagem dos autores, a ferramenta destacada por sua importância são os oito princípios evolutivos, uma vez que são capazes de estabelecer uma previsão de evolução tecnológica.

Dadas as conclusões dos autores, é apresentado o framework 'Uso, Usabilidade e Significado' (SANDERS, 1992) onde, para cada decisão de predomínio no enfoque no DNP, eles concluem que uma abordagem entre DCSH ou TRIZ é mais apropriada. Quando o objetivo é focar no uso do produto, os problemas são de cunho técnico, e consequente o uso das ferramentas TRIZ tornam-se fundamentais. Caso o enfoque seja no significado do produto, entender o que os usuários desejam, quais seus valores, comportamentos, expectativas, etc., passa a ser prioridade, resultando numa necessidade de ênfase no DCSH. Um caso especial encontra-se quanto deseja-se focar na usabilidade. Como este aspecto refere-se à facilidade como determinado produto é usado, é importante considerar um feedback dos usuários, e posteriormente apostar na metodologia da TRIZ para resolver problemas provenientes dessas informações. Deste modo, para resolver problemas de usabilidade é aconselhado combinar as abordagens tanto da TRIZ quanto do DCSH.

Em acordo com as conclusões de Pelt e Hey (2011), outros trabalhos também tratam com prioridade a atenção nas demandas dos usuários, mas sem esquecer o fator inovativo fundamental no ambiente altamente competitivo no mercado de desenvolvimento de produtos. Dentro deste contexto, uma das ferramentas mais utilizadas é a QFD. No subcapítulo a seguir são apresentadas as pesquisas encontradas que versam sobre a integração da QFD e TRIZ.

\subsection{TRIZ e o Método de Desdobramento da Função Qualidade}

Em um cenário cujas demandas de qualidade e performance são muito importantes para os usuários, a QFD é um importante método capaz de traduzir as demandas dos clientes em requisitos de qualidade (LOOS; AUGUSTO; MIGUEL, 2014). Dado este aspecto importante da QFD, cujo enfoque é justamente um ponto onde metodologia da TRIZ não prioriza, a integração destas duas metodologias, proposta por Yamashina, Ito e Kawada (2000), foi realizada na criação de um novo método chamado IPDP (Processo de Desenvolvimento de Produtos Inovativos, na sigla em inglês).

O desenvolvimento do IPDP utiliza a ferramenta HOC (Casa da Qualidade, na sigla em inglês) da QFD para converter as informações do mercado em requisitos dos usuários e, partindo disso, é usada como uma importante ferramenta no desenvolvimento do conceito do produto no processo de planejamento. Foram retiradas da TRIZ, visando a integração ao IPDP, algumas de suas ferramentas, tais como os princípios para resolução de problemas contraditórios, buscando resolver 
problemas de conflito nos requisitos dos usuários, e também o método de análise campo-substância para verificar problemas de interação entre componentes e suas possíveis soluções. Por fim, o método apresentado indica um algoritmo para derivar outras novas inovações tecnológicas, às quais são aplicadas funções de avaliação para determinar quais as soluções finais. Assim, portanto, podese que o IPDP abrange os processos desde o planejamento do produto até o design conceitual.

Dentre os principais méritos do IPDP destaca-se seu potencial na geração de novas ideias tecnológicas graças a TRIZ, etapa para a qual eles sugerem a criação de duas árvores, sendo uma para mapear os mecanismos, e outra para mapear as funções. A ferramenta proposta, baseada na análise campo-substância, é capaz de remover a dependência da intuição e experiência do designer através da sistematização do método do IPDP.

Seguindo a mesma base de Yamashina, Ito e Kawada (2000) quanto a importância das demandas dos consumidores, Huang (2013) também desenvolveu seu trabalho integrando a matriz de planejamento de produtos Casa da Qualidade com a TRIZ. O objetivo também é o mesmo: utilizar a HOC para encontrar as especificações necessárias para o produto objetivando aumentar sua fatia de mercado, e depois utilizar as ferramentas da TRIZ para auxiliar na solução dos difíceis problemas técnicos que surgem da necessidade de inovação. Seguindo este raciocínio, foi proposto um passoa-passo para o processo de DNP de acordo com o processo QFD, através do qual buscam assegurar que os desejos dos consumidores serão atendidos. Os passos são apresentados no Quadro 3.

Quadro 3 - Passo-a-passo integrando a matriz de planejamento de produtos Casa da Qualidade e a TRIZ

Passo-a-passo para o DNP integrando a TRIZ e o QFD

Passo 1:

Planejamento do produto: justificativa, pesquisa e análise, análise de concorrentes, necessidade de mercado, oportunidades tecnológicas (TRIZ como poderosa ferramenta na previsão tecnológica), etc.;

Passo 2: Pesquisa sobre necessidades dos usuários com base na Lei da Idealidade;

Passo 3: Compreender e antecipar as necessidades dos consumidores através do modelo de Kano (identificar as necessidades básicas, as necessidades de performance e encontrar os fatores surpresa);

Passo 4: Avaliar a importância das necessidades dos consumidores;

Utilizar a HOC para verificar quais as características mais desejadas para descobrir quais mecanismos necessitam melhoras. Nesta mesma etapa, utiliza-se a TRIZ para prever as melhorias tecnológicas com base

Passo 5: na Lei da Idealidade com objetivo de otimizar o custo, preço e performance, e utilizar a matriz de contradição para resolver problemas e conflitos;

Passo 6: Geração e seleção de conceitos, etapa na qual a TRIZ ajuda no desenvolvimento e refino da solução.

Desenvolvimento das partes, na qual a análise funcional da TRIZ exerce função importante na priorização

Passo 7: do design;

Passo 8: Planejamento do processo de manufatura;

Passo 9: Controle do produto, fase onde utiliza-se os bancos de dados da TRIZ para verificar meios de melhorar os processos de manufatura. Ou seja, é uma etapa que pensa na melhoria do processo de manufatura;

Teste e validação, etapa onde são feitas verificações para avaliar se o produto apresenta a qualidade e

Passo 10: Teste e validação, etapa onde são feitas

Passo 11: Lançamento no mercado, onde desenvolve-se ações mercadológicas e suporte técnico.

Fonte: adaptado de Huang (2013)

Seguindo a linha cujo objetivo é atender os desejos dos consumidores e do mercado, Liu e Cheng (2016) focaram sua pesquisa em aumentar a certeza e completude das informações coletadas nas pesquisas que devem ser realizadas para estabelecer os requisitos desejados. Por 
estes motivos, desenvolvem uma nova Função de Desdobramento da Função Qualidade baseada nos números Grey com a integração das técnicas da TRIZ.

Os números grey são usados para descrever uma determinada informação incerta e ajudar em tomadas de decisão (XIE; LIU, 2010). Através do método de ranqueamento de grey e na QFD, Liu e Cheng (2016) propõem um método que calcula a importância dos requisitos dos consumidores e depois os traduz em parâmetros de engenharia, que por sua vez são calculados, também através do método de ranqueamento de grey, suas importâncias. De posse desses valores calculados, encontra-se o 'grau de greyness', que nada mais é que uma medição do nível de incerteza dos valores encontrados, o que permitirá ranquear os parâmetros de engenharia por ordem de importância. Dadas essas adaptações da QFD com a TRIZ, a próxima etapa é aplicar a matriz de contradição priorizando os parâmetros que apresentarem maior peso negativo. A este método foi dado o nome de GQFD (Grey Quality Function Deployment).

A utilização da TRIZ e QFD integradas, porém, não foi a única proposta de adaptação metodológica encontrada. A associação com outros métodos como, por exemplo o Raciocínio Baseado em Casos (CBR, na sigla em inglês), também apresentou estudos, todos relativos ao desenvolvimento de produtos sustentáveis e ecologicamente amigáveis. Apesar disso, a QFD também apresenta suas aplicações neste segundo campo.

\subsection{TRIZ, o Desenvolvimento de Produtos Ecológicos, CBR e a ECQFD}

Apesar de incluir questões conscientes quanto a utilização dos recursos, a TRIZ não apresenta uma finalidade 'verde', o que atualmente apresenta-se como fundamental na concepção de novos produtos. Portanto, afirmando que não foram encontradas abordagens que considerem a sustentabilidade em cada etapa do processo de desenvolvimento dos produtos, Russo, Regazzoni e Montecchi (2011) buscaram estabelecer diretrizes capazes de avaliar e melhorar os produtos e seus processos através de uma perspectiva ecológica com o uso dos fundamentos da TRIZ.

Neste contexto de ausência de orientações para um projeto de produto ecologicamente amigável, as ferramentas da TRIZ adotadas foram, especialmente, as 8 tendências evolutivas e os fundamentos acerca dos recursos, além da busca pela idealidade. Estas diretrizes buscam avaliar sistemas de inovação ecológica de uma maneira mais eficiente, estabelecendo, para cara orientação, determinadas regras a serem seguidas (Tabela 1).

Seguindo as metas de melhorar o desenvolvimento de produtos dentro de um contexto ecológico, Yang e Chen (2011) apresentam a integração da CBR e da TRIZ. Sua proposta parte do princípio que a CBR, já utilizada no design ecológico, é utilizada para elaborar estratégias de descarte, reciclagem, avaliação do custo-benefício e do gerenciamento ambiental, enquanto a TRIZ apoia na busca por um maior nível de inovação. Neste ponto Yang e Chen apresentam uma série de trabalhos que já utilizam a TRIZ como meio de atingir objetivos 'verdes', mas que adotam apenas aplicações das ferramentas em estudos de caso, sem propor alguma metodologia específica.

As ferramentas da TRIZ, combinadas com a CBR, foram a matriz de contradições e os 40 princípios inventivos, os padrões evolutivos e a busca pela idealidade dos sistemas, as quais foram adotadas para elevar a criatividade de um modelo proposto para garantir que o designer consiga atingir seu objetivo com facilidade. Nesta abordagem, antes da utilização destas ferramentas, analisando de modo análogo, a CBR substitui a $\mathrm{HOC}$ no início do processo de projeto, quando busca as funções desejadas. A integração de ambos métodos é explicada pelas seguintes etapas: (i) criação 
de uma base de dados com produtos indexados de acordo com todas suas funções; (ii) processos de recuperação, reuso e revisão das informações coletadas nos bancos de dados. Uma avaliação de similaridade deve ser feita (estas duas primeiras instruções resumem os métodos CBR adotados); (iii) seleção de elementos de design ecológico com vistas a diminuir o impacto ambiental. Neste ponto eles sugerem o uso dos 7 elementos ecologicamente amigáveis da WBCSD (World Business Council for Sustainable Development) apresentados na Quadro 4; (iv) utilizar as ferramentas da triz para melhorar as características funcionais.

Tabela 1 - Eco-diretrizes e regras

\begin{tabular}{|c|c|c|c|c|}
\hline & & Diretrizes & & Regras \\
\hline \multirow{2}{*}{ 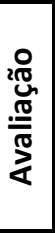 } & G1 & Modelagem do sistema & $\begin{array}{l}\mathrm{R} 1.1 \\
\mathrm{R} 1.2\end{array}$ & $\begin{array}{l}\text { Identificação da função principal } \\
\text { Descrição física }\end{array}$ \\
\hline & $\mathrm{G} 2$ & Avaliação dos recursos & $\begin{array}{l}\text { R2.1 } \\
\text { R2.2 } \\
\text { R2.3 }\end{array}$ & $\begin{array}{l}\text { Índices de exploração dos recursos } \\
\text { Análise das condições do sistema no presente/passado } \\
\text { Identificar recursos externos }\end{array}$ \\
\hline \multirow{6}{*}{ 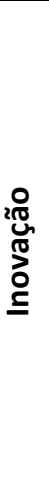 } & G3 & Economia de recursos & $\begin{array}{l}\text { R3.1 } \\
\text { R3.2 } \\
\text { R3.3 }\end{array}$ & $\begin{array}{l}\text { Usar os conceitos da idealidade } \\
\text { Reduzir conversão de energia à zero } \\
\text { Explorar outras tecnologias }\end{array}$ \\
\hline & G4 & Interação dos componentes & $\begin{array}{l}\mathrm{R} 4.1 \\
\mathrm{R} 4.2\end{array}$ & $\begin{array}{l}\text { Fazer as ações ressonarem } \\
\text { Coordenar os campos }\end{array}$ \\
\hline & G5 & Dinamização do sistema & R5.1 & Dinamizar o sistema \\
\hline & G6 & Simplificação do sistema & $\begin{array}{l}\mathrm{R} 6.1 \\
\mathrm{R} 6.2\end{array}$ & $\begin{array}{l}\text { Eliminar componentes não úteis } \\
\text { Resolver contradições }\end{array}$ \\
\hline & G7 & $\begin{array}{l}\text { Exploração de recursos } \\
\text { externos }\end{array}$ & $\begin{array}{l}\text { R7.1 } \\
\text { R7.2 }\end{array}$ & $\begin{array}{l}\text { Unir sistemas técnicos } \\
\text { Evoluir para super-sistema }\end{array}$ \\
\hline & G8 & Campos cooperativos & R8.1 & Aumentar o envolvimento campo-substância \\
\hline
\end{tabular}

Fonte: adaptado de Russo, Davide, Regazzoni e Montecchi (2011)

Quadro 4 - Metas ecológicas da WBCSD

\begin{tabular}{ll}
\hline & \multicolumn{1}{c}{ Metas ecológicas } \\
\hline A. & Reduzir quantidade de materiais \\
B. & Reduzir o consumo de energia \\
C. & Reduzir a dispersão de gases e materiais tóxicos \\
D. Aumentar a reciclabilidade dos materiais \\
E. Maximizar o uso de materiais sustentáveis e renováveis \\
F. Aumentar a durabilidade dos produtos \\
G. Aumentar a oferta de serviços nos produtos
\end{tabular}

Fonte: adaptado de Yang e Chen (2011)

O trabalho demonstra que o CBR é capaz de obter, eficientemente, as funções características desejadas. A partir disso o método da TRIZ, que suporta diferentes campos da tecnologia para elevar a criatividade do modelo, garante que o designer possa alcançar o objetivo com facilidade. Neste ponto a adoção dos elementos eco-amigáveis do WBCSD ajuda a definir os objetivos da inovação ecológica. Assim, Yang e Chen (2012) realizaram novo estudo que agrega a matriz do método LCA (Avaliação do Ciclo de Vida, sigla em inglês) para avaliar o impacto do produto no meio ambiente.

A matriz adotada por Yang e Chen (2012) foi desenvolvida por Graedel, Allenby e Comrie 
(1995), utilizada pela AT\&T, é capaz de avaliar o ciclo de vida de um produto de modo eficiente. Esta matriz relaciona os estágios de vida do produto com tópicos ambientais como a escolha do material, gasto de energia, resíduos sólidos, líquidos e gasosos, aos quais atribuem-se valores de 0 (maior impacto) a 4 (menor impacto) nas situações presentes e passadas do produto. Através da soma de todos os valores, que pode chegar ao máximo de 100, é possível comparar se a solução proposta é melhor, equivalente ou pior que a original.

A partir do desenvolvimento dos dois trabalhos de Yang e Chen $(2011,2012)$ é possível reforçar a importância e impacto que a base de dados causa ao framework da CBR em obter uma solução. Por outro lado, a TRIZ reforça-se como um método poderoso e eficiente capaz de identificar e encontrar soluções que facilitam e melhoram o design de produtos ecologicamente amigáveis.

Criando uma abordagem cuja perspectiva pondera relações ecológicas com mercadológicas, Vinodh, Kamala e Jayakrishna (2014) propõem integrar TRIZ, ECQFD (Desdobramento da Função Qualidade Ambientalmente Consciente, na sigla em inglês) e AHP (Processo de Análise Hierárquica, na sigla em inglês). A ECQFD apresenta passos que buscam identificar os desejos dos consumidores a nível ambiental, identificar as características técnicas e de engenharia a nível ambiental, e identificar alvo para melhoramento. O método ainda apresenta meio de avaliar os impactos causados pelas soluções através da matriz LCA (VINODH; RATHOD, 2010).

De acordo com todos os dados coletados pela ECQFD, Vinodh, Kamala e Jayakrishna (2014) adotam a matriz de contradições da TRIZ para resolver os conflitos que emergentes através dos princípios inventivos e os parâmetros de engenharia. Como meio de avaliar qual a melhor solução dentre as encontradas, utiliza-se um AHP baseado nos múltiplos critérios definidos no Quadro 5.

Quadro 5-Os múltiplos critérios utilizados na AHP para avaliar
\begin{tabular}{l} 
Critérios para a análise hierárquica \\
\hline A. Vida útil do produto \\
B. Inovação \\
C. Emissões de carbono \\
D. Atratividade no mercado \\
E. Descarte ao final da vida útil \\
F. Capacidade tecnológica \\
G. Peso \\
H. Oferta
\end{tabular}

Fonte: adaptado de Vinodh, Kamala e Jayakrishna (2014)

\subsubsection{O Método iTree}

Russo, dando continuidade a seu trabalho, juntamente com Rizzi e Motelisciani (2014), buscou considerar a dúvida dos designers quando considerando as questões de sustentabilidade, uma vez que este tipo de abordagem apresenta limitações em sua aplicação. Aliada a esta dificuldade, estão outros problemas como a necessidade de se apresentar grande comprometimento no projeto, conhecimento da equipe, e restrições devido à pouca possibilidade de variação no design. Visando mitigar esses problemas, os autores apresentam o iTree. O método consiste no uso de um framework estruturado baseado nas ecológicas desenvolvidas com origem da TRIZ (RUSSO; REGAZZONI; MONTECCHI, 2011). Resumidamente, o método busca integrar à TRIZ a questão do ciclo de vida do produto usando LCA através de uma nova matriz. 
A matriz, elaborada para o método iTree, relaciona ferramentas que podem ser aplicadas visando um objetivo ecológico (reduzir, mudar ou repor/evitar) levando em consideração os ciclos de vida (pré-manufatura, manufatura, uso e fim da vida útil). Estas ferramentas adotadas, cuja origem remete diretamente à TRIZ, são os princípios inventivos e os parâmetros de engenharia (derivando-se, portanto, a matriz de contradição), as soluções padrões, as tendências de evolução tecnológica, os fundamentos sobre recursos e também um método integrado da TRIZ com FMEA (Failure Mode and Effect Analysis) desenvolvido por Regazzoni e Russo (2011). Este método integrado, por sua vez, apresenta ferramentas da TRIZ relacionadas à avaliação dos recursos, análise campo-substância e as soluções padrões. O objetivo é facilitar e diminuir a necessidade de alto grau de expertise na busca pela redução a ocorrência de falhas através da antecipação de problemas e soluções técnicas (REGAZZONI; RUSSO, 2011).

Resumidamente, as diretrizes do método compreendem em reconhecer a zona crítica que deve ser identificada através de uma avaliação por LCA. A partir disso a matriz iTree relaciona 17 objetivos ecológicos com 47 ferramentas em uma matriz com cerca de 300 orientações. O resultado contribui no delineamento de uma estratégia para combater as questões eminentes da análise LCA.

\subsection{TRIZ e o DFSS}

A DFSS (Design for Six Sigma) tem objetivo de aumentar a competitividade de produtos no mercado. Sua abordagem estruturada é usada na solução de problemas baseados na melhora ou otimização de produtos ou processos existentes. Busca-se traduzir expectativas dos clientes e implementar a alternativa mais eficiente, verificando se o produto comporta normas ambientais ao qual se aplica. Assim pode-se dizer que sua metodologia é muito semelhante a ECQFD.

A metodologia da DFSS compreende 5 fases: definição, medida, análise, design e verificação. A aplicação da TRIZ, de acordo com a sugestão dos autores, se dá na fase de análise. As bases utilizadas da TRIZ são os parâmetros de engenharia para identificar os conflitos técnicos, a utilização dos 40 princípios inventivos para resolver as contradições, e a Análise Campo-Substância para modelar problemas técnicos e derivar soluções. O Quadro 6 resume a metodologia proposta.

\section{Quadro 6 - Resumo das 5 fases da metodologia do DFSS com a utilização da TRIZ}

\section{Resumo das fases da DFSS integrando a TRIZ}

1. Fase de definição: determinação das responsabilidades de cada membro do time de projeto, assim como os objetivos do projeto. Definir os requisitos dos consumidores;

2. Fase de medição: tradução da voz dos consumidores em requisitos críticos através de ferramentas de medição. Fase de análise: utiliza-se as ferramentas TRIZ (IFR, recursos, contradição, IFM, método de Pugh) para gerar os conceitos para produtos e peças. Ainda que não seja utilizado pelo autor, um trabalho que incorpora a metodologia da TRIZ e o método de Pugh, que é utilizado para estabelecer um critério lógico e significativo para definir a melhor ideia encontrada para apoiar as estratégias de fabricação, pode ser encontrada em 'TRIZPUGH Model, New Approach for Creative Problem Solving and Decision Making (KARNJANASOMWONG; THAWESAENGSKULTHAI, 2015).

Fase de design: usar DOE (Design of Experiments, cujo método constitui na alteração de uma variável

4. independente, ou fator de entrada, para observar as mudanças na variável dependente, ou saída do sistema) para testar e selecionar o design final.

5. Fase de verificação: serve para assegurar a efetividade das mudanças da última fase. 


\section{Conclusões e Considerações Finais}

A TRIZ é, notoriamente, uma teoria com muito potencial para identificação de problemas inovativos e inventivos, e especialmente muito capaz de auxiliar na busca por soluções. Dentre as ferramentas mais utilizadas nos trabalhos pesquisados estão a matriz de contradição, seguido dos oito princípios evolutivos e, logo após, da análise campo-substância.

De todos os trabalhos pesquisados é possível perceber que a TRIZ apresenta alguns pontos fracos, a notar a ausência da abordagem com enfoque nas demandas dos usuários e do mercado. Este último, nos dias atuais, é um fator muito influenciado por uma tendência à popularização do design ecológico e sustentável, o que é outro fator cuja abordagem na TRIZ encontra-se apenas em termos do aproveitamento racional dos recursos. Buscando resolver estes problemas, foram desenvolvidas pesquisas que propõem e apresentam metodologias, algoritmos e diretrizes, todas elas utilizando a TRIZ como um pilar fundamental no design de novos produtos.

Cumprindo o objetivo desta pesquisa, destacam-se as linhas de pesquisas que integram a TRIZ apresentadas no Quadro 7.

Quadro 7: linhas de pesquisa que integram a TRIZ

\section{Linhas de pesquisa englobando a TRIZ}

a. TRIZ e o DCSH como meios de resolver problemas relacionados aos desejos e necessidades dos usuários;

b. TRIZ integrada à QFD buscando solucionar problemas contraditórios na tradução dos requisitos de projeto;

c. Resolução de problemas de performance e qualidade encontrados através da HOQ da QFD através da TRIZ;

d. Utilização da TRIZ em relação à dificuldade de design de produtos ecologicamente amigáveis;

e. Aproveitamento das bases de dados da CBR tanto para maximização das soluções TRIZ;

f. Integração da TRIZ à métodos de avaliação de impactos ambientais;

g. Integração da TRIZ com métodos de tomada de decisão na escolha das opções.

h. Incorporação da TRIZ a metodologias de projetos de produto.

\section{Fonte: o autor}

\section{Sugestões para Futuras Pesquisas}

Durante a pesquisa sobre a TRIZ, foi possível notar que se trata de uma ferramenta sistemática muito poderosa na resolução de problemas inventivos. É notável, porém, que sua associação às metodologias de projeto é capaz de maximizar seu potencial inventivo. Uma compilação dos pontos fortes dos métodos capazes de levar em consideração questões ambientais, mercadológicas e dos usuários poderia ser de grande valia na busca por uma metodologia geral para desenvolvimento de produtos centrados nos usuários e no meio ambiente com alto poder de geração de soluções e inovação. A reunião, por exemplo, dos bancos de dados da CBR combinada com a QFD e a TRIZ poderiam alavancar a identificação de problemas e possibilidades de inovação.

Outro ponto com potencial exploratório é mudança da ótica nas pesquisas utilizando a TRIZ. Muito utilizada para adaptar ou integrar metodologias, sua força como uma abordagem sistemática completa na resolução de problemas poderia torna-la ponto de partida de novas pesquisas, à qual seria desenvolvida e acrescentada novas abordagens referentes aos seus pontos fracos destacados anteriormente. 


\section{Referências}

ALTSHULLER, G. Creativity as an exact science: The theory of the solution of inventive problems. Journal of development economics, v. 91, n. 1, p. 64-76, 1984.

ALTSHULLER, G. And Suddenly the Inventor Appeared: TRIZ, the Theory of Inventive Problem Solving. 2nd. ed. Technical Innovation Center, Inc., 1996.

ALTSHULLER, G. S.; SHULYAK, L.; RODMAN, S. 40 Principles: TRIZ Keys to Technical Innovation. 1st. ed. Technical Innovation Center, Inc., 1997.

BRAD, S. et al. TRIZ to Support Blue-design of Products. Procedia CIRP, v. 39, p. 125-131, 2016.

CHANG, Y. S. et al. Effect of TRIZ on the creativity of engineering students. Thinking Skills and Creativity, v. 19, p. 112-122, 2016.

CHECHURIN, L. TRIZ in Science: Reviewing Indexed Publications. Procedia CIRP, v. 39, p. 156-165, 2016.

CHEN, L.-S.; CHEN, S.-H. Using TRIZ Techniques to New Product Function Development of Smart Phones. IEMS, v. 10, n. 3, p. 179-184, 2011.

CONG, H.; TONG, L. H. Grouping of TRIZ Inventive Principles to facilitate automatic patent classification. Expert Systems with Applications, v. 34, n. 1, p. 788-795, 2008.

DOMB, E.; MILLER, J.; MACGRAN, E. The Seventy-Six Standard Solutions: How They Relate to the 40 Principles of Inventive Problem Solving. The TRIZ Journal, 1999.

GRAedel, T. E.; ALlenbY, B. R.; COMRIE, V. R. Matrix Approaches to Abridged Life Cycle Assessment. Environmental Science and Technology, v. 29, n. 3, p. 134-139, 1995.

HUANG, F. Technology innovation and new product development process integrating QFD and TRIZ. 2013 Suzhou-Silicon Valley-Beijing International Innovation Conference, p. 127-131, 2013.

IDEATION INTERNATIONAL INC. Tools of Classical TRIZ. Ideation International Inc., 1999.

LANGEVIN, R.; TECHNICAL INNOVATION CENTER INC. Ideality. Disponível em: <http://www.triz.org/triz/ideality>.

LEON, N. Trends and patterns of evolution for product innovation. The TRIZ Journal, 2006.

LIU, H. T.; CHENG, H. S. An improved grey quality function deployment approach using the grey TRIZ technique. Computers and Industrial Engineering, v. 92, p. 57-71, 2016.

LOOS, M. J.; AUGUSTO, P.; MIGUEL, C. Utilização do QFD no desenvolvimento de novos produtos: uma análise das publicações em periódicos nacionais. v. 15, p. 17-31, 2014.

LÓPEZ, J.; ALMEIDA, R. L. DE; ARAUJO-MOREIRA, F. M. TRIZ: criatividade como uma ciência exata? Revista Brasileira de Ensino de Física, v. 27, n. 2, p. 205-209, 2005.

MISHRA, U. The Concept of Resources in TRIZ. 2013.

PELT, A. VAN; HEY, J. Using TRIZ and human-centered design for consumer product development. Procedia Engineering, v. 9, p. 688-693, 2011.

PIMENTEL, A. R. Considerações sobre TRIZ e sua aplicação no desenvolvimento de software. p. 15, 2013. 
REGAZZONI, D.; RUSSO, D. TRIZ tools to enhance risk management. Procedia Engineering, v. 9, p. 40-51, 2011.

RUSSO, D.; REGAZZONI, D.; MONTECCHI, T. Eco-design with TRIZ laws of evolution. Procedia Engineering, v. 9, p. 311-322, 2011.

RUSSO, D.; RIZZI, C.; MONTELISCIANI, G. Inventive guidelines for a TRIZ-based eco-design matrix. Journal of Cleaner Production, v. 76, p. 95-105, 2014.

SALAMATOV, Y. The Right Solution at the Right Time: A Guide to Innovative Problem Solving. Insytec BV., 1999.

SANDERS, E. Converging Perspectives: Product Development Research for the 1990s. Design Management Journal, v. 3, n. 4, p. 49-54, 1992.

SAVRANSKY, S. D. Engineering of Creativity: Introduction to TRIZ Methodology of Inventive Problem Solving. Boca Raton: CRC Press LLC, 2000.

STAROVOYTOVA, D. ET. A. Theory of inventive problem solving (TRIZ): his-story. IJISET International Journal of Innovative Science, Engineering \& Technology, v. Vol. 2, n. Issue 7, p. 8695, 2015.

VINODH, S.; KAMALA, V.; JAYAKRISHNA, K. Integration of ECQFD, TRIZ, and AHP for innovative and sustainable product development. Applied Mathematical Modelling, v. 38, n. 11-12, p. 2758-2770, 2014.

VINODH, S.; RATHOD, G. Integration of ECQFD and LCA for sustainable product design. Journal of Cleaner Production, v. 18, n. 8, p. 833-842, 2010.

WANG, F.-K.; YEH, C.-T.; CHU, T.-P. Using the design for Six Sigma approach with TRIZ for new product development. Computers \& Industrial Engineering, v. 98, p. 522-530, 2016.

XIE, N. MING; LIU, S. FENG. Novel methods on comparing grey numbers. Applied Mathematical Modelling, v. 34, n. 2, p. 415-423, 2010.

YAMASHINA, H.; ITO, T.; KAWADA, H. Innovative product development process by integrating QFD with TRIZ. Seimitsu Kogaku Kaishi/Journal of the Japan Society for Precision Engineering, v. 66, n. 11, p. 1705-1710, 2000.

YANG, C. J.; CHEN, J. L. Accelerating preliminary eco-innovation design for products that integrates case-based reasoning and TRIZ method. Journal of Cleaner Production, v. 19, n. 9-10, p. 998-1006, 2011.

YANG, C. J.; CHEN, J. L. Forecasting the design of eco-products by integrating TRIZ evolution patterns with CBR and Simple LCA methods. Expert Systems with Applications, v. 39, n. 3, p. 28842892, 2012. 